\title{
Synchronization Study and Introductory Experiment of Laser-Triggered Surface Flashover in Voltage Pulse
}

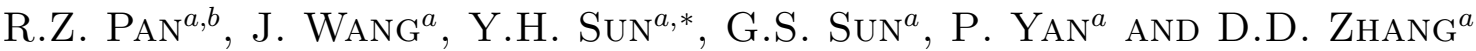 \\ ${ }^{a}$ Institute of Electrical Engineering, Chinese Academy of Sciences \\ Beijing, 100190, China \\ ${ }^{b}$ Graduate School of Chinese Academy of Sciences, Beijing, 100049, China
}

\begin{abstract}
With the aim of studying the characteristics of laser-triggered surface flashover in voltage pulse, synchronization problem of laser pulse and voltage pulse should be solved. A single/double harmonic, with wavelength $\lambda$ of $1064 / 532 \mathrm{~nm}$, Q-switched Nd:YAG laser is used to trigger the surface flashover. The synchronization problem is solved using a self-made digital delay/pulse generator. The delay time and jitter of Marx's trigger input and output, Marx's trigger output and Marx's output, laser input and output are respectively measured. Based on the result of the delay time and the timing sequence of the laser triggering system, the synchronization of laser pulse and voltage pulse is obtained through adjusting the channel delay time of digital delay/pulse generator. In addition, introductory experiment of laser-triggered surface flashover is carried out using the flat electrodes and columned insulators.
\end{abstract}

PACS numbers: 52.80.-s, 51.50.+v

\section{Introduction}

Fast closing switch $[1,2]$ is one of the most important component in pulsed power technology, and it is also one of the bottlenecks of pulsed power development. Surface flashover switch [3] can provide many advantages including simplicity of construction, accurate trigger control, low switching jitter and wide dynamic operating range. The delay time and jitter of laser-triggered surface flashover switch are less than that in electric-triggered surface flashover switch [4-7]. The former can be applied in parallel-pulsed power system to solve the synchronization problem hopefully. Fast low-jitter closing switch is one of the schemes to realize accelerator - dielectric wall accelerator (DWA) [8], so laser-triggered surface flashover switch can impel the development of DWA hopefully.

This paper studies the delay time and jitter of signals through experiment, and obtains exact synchronization of laser pulse and voltage pulse controlled by self-made digital delay and pulse generator. In addition, introductory experiment of laser triggered surface flashover is carried out using the flat electrodes and columned insulators.

\section{Experiment platform and synchronization}

Experiment platform of laser-triggered surface flashover is shown in Fig. 1. 3-channel Digital delay/pulse generator controls the laser's Xe lamp and

\footnotetext{
* corresponding author; e-mail: yhsun@mail.iee.ac.cn
}

Marx's trigger. Marx's trigger triggers Marx generator, then Marx generator delivers high voltage pulse. When oscillograph detects the high voltage pulse, the TTL output can produce a TTL voltage pulse to trigger the laser's Q-switch. The high voltage pulse and laser pulse can be exerted synchronously on the test sample. Oscillograph in experiment is Agilent 54832B DSO.

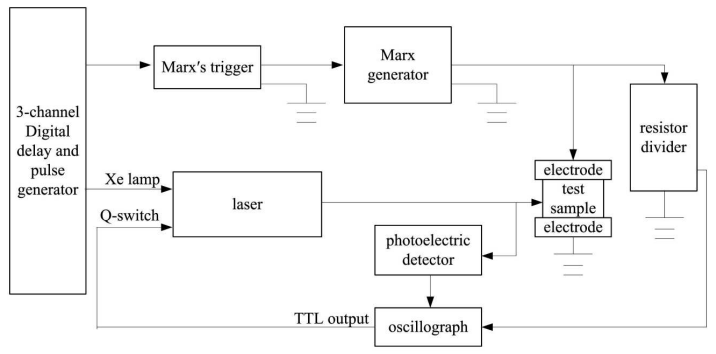

Fig. 1. Schematic diagram of experiment system.

Voltage waveform of Marx's trigger is shown in Fig. 2. Narrow pulse at the front of voltage waveform is caused by the gap connecting with the Marx trigger. CH1 waveform is the output voltage of 3-channel digital delay and pulse generator, and $\mathrm{CH} 2$ waveform is the output voltage of Marx's trigger (Fig. 2). CH2 waveform is measured by Tektronix P6015 A high voltage probe. Delay time and jitter of Marx's trigger can be obtained through the experiment, the delay time and the jitter are $21.109 \mu$ s and $2.289 \mu \mathrm{s}$, respectively.

The output waveform of the Marx generator is shown in Fig. 3. CH1 waveform is the output voltage of the 


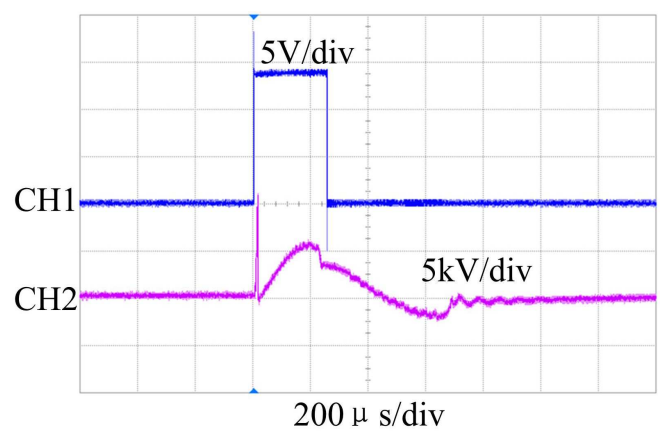

Fig. 2. Marx's trigger.

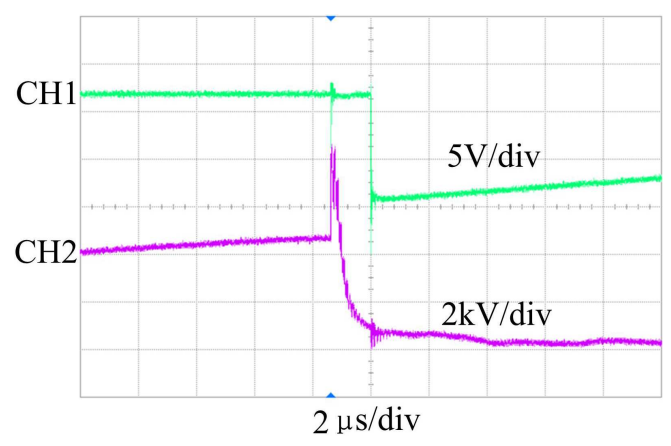

Fig. 3. Marx generator.

Marx generator, and $\mathrm{CH} 2$ waveform is the output voltage of Marx's trigger (Fig. 3). CH1 is measured by the resistor divider, with a ratio of 1850 and $\mathrm{CH} 2$ waveform is measured by Tektronix P6015 A high-voltage probe. The output voltage of Marx generator is $31 \mathrm{kV}$, and the rise time of the voltage is less than $20 \mathrm{~ns}$. Delay time and jitter of the Marx generator can be obtained through the experiment, the delay time and the jitter are $992.9 \mathrm{~ns}$ and $436.3 \mathrm{~ns}$, respectively.

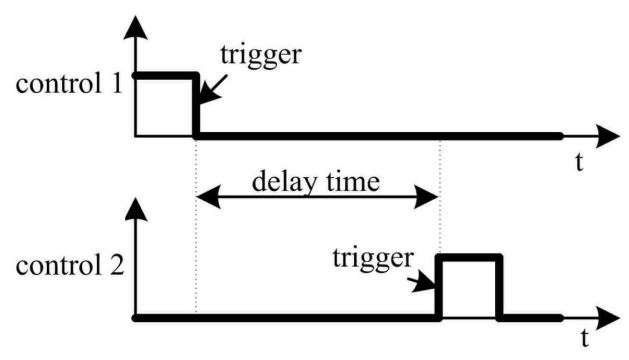

Fig. 4. Timing sequence of laser.

The control timing sequence of SGR-S400 laser is shown in Fig. 4. Control 1 is the control signal of the laser's Xe lamp; control 2 is the control signal of the laser's Q-switch; and the delay time is $200 \mu \mathrm{s}$. Then, the laser outputs the pulse. 3-channel digital delay and pulse generator triggers the laser's Xe lamp and Q-switch

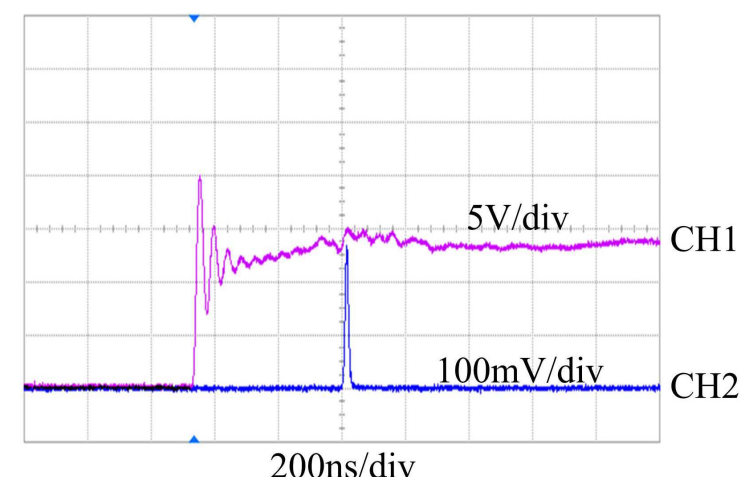

Fig. 5. Laser pulse and control signal.

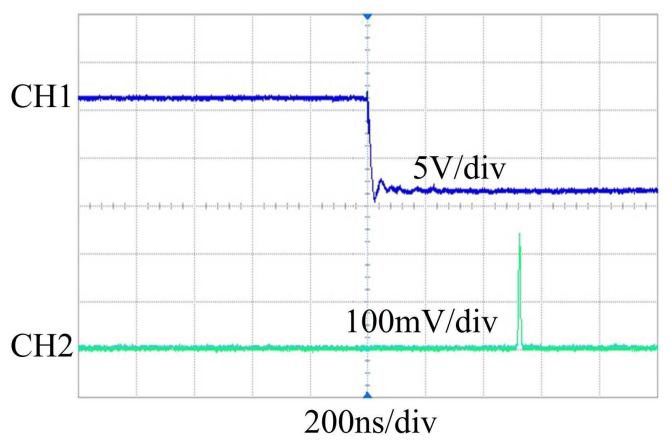

Fig. 6. Synchronization waveforms.

with the control timing sequence in Fig. 4. The pulse of laser and control signal of Q-switch measured through experiment are shown in Fig. 5. CH1 waveform is control signal of Q-switch, and $\mathrm{CH} 2$ waveform is the pulse output of the laser. CH2 waveform is measured by Thorlabs DET210 photoelectric detector. Delay time and jitter of Q-switch's control signal and laser pulse can be obtained through the experiment, among which delay time is $475.5 \mathrm{~ns}$ and jitter is $4.182 \mathrm{~ns}$, respectively.

Based on the control timing sequence of the laser, the delay time of the first channel and the second channel of digital delay/pulse generator is initialized with $228 \mu$ s. The first channel controls Xe lamp of the laser, and the second channel controls the Marx trigger. The synchronization wave of laser pulse and voltage pulse is shown in Fig. 6. CH1 waveform is the output voltage of the Marx generator, and $\mathrm{CH} 2$ waveform is the output laser pulse of the laser. Delay time and jitter of Marx generator's voltage pulse and laser pulse can be obtained through the experiment, among which delay time is $516.1 \mathrm{~ns}$ and jitter is $4.5 \mathrm{~ns}$, respectively.

\section{Introductory experiment}

With experiment platform, laser-triggered surface flashover is experimented. The material of electrode is stainless steel and the electrode diameter is $100 \mathrm{~mm}$; the 


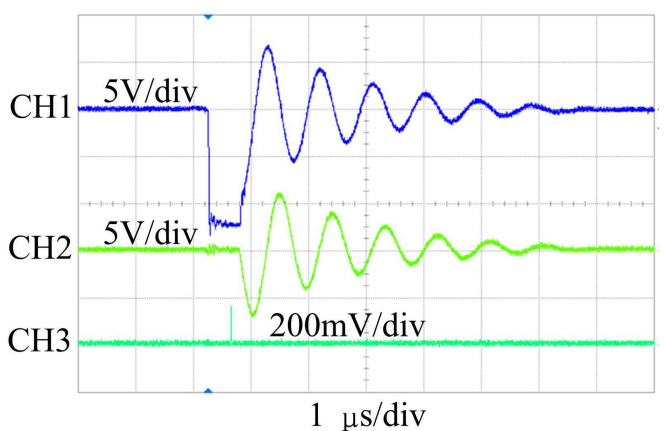

Fig. 7. Test waveforms.

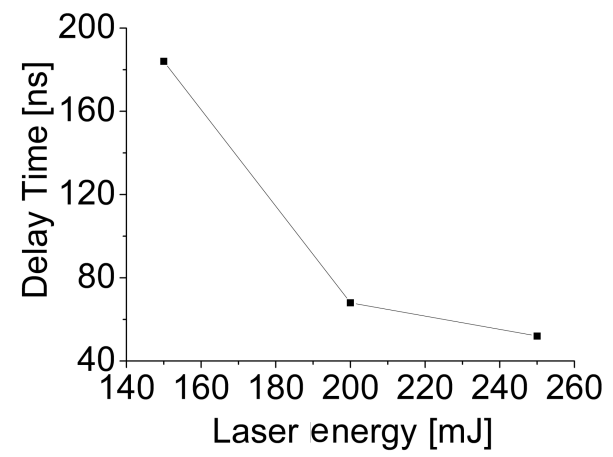

Fig. 8. Delay time and laser energy.

material of test sample is nylon and the diameter and thickness of the sample are $40 \mathrm{~mm}$ and $8 \mathrm{~mm}$, respectively, and laser pulse's wavelength is $532 \mathrm{~nm}$ with $6 \mathrm{~mm}$ diameter. Flashover waveforms are shown in Fig. 7. CH1 waveform is the voltage of flashover; $\mathrm{CH} 2$ waveform is the current of flashover measured with the Rogowski coil of 200 ratios; and $\mathrm{CH} 3$ waveform is the output laser pulse of the laser. The self-flashover voltage of nylon is $24 \mathrm{kV}$, and the voltage at sample is $22 \mathrm{kV}$ in laser-triggered surface flashover experiment. The delay time and jitter with laser energy are shown in Figs. 8 and 9, respectively. Delay time and jitter decrease with the increase of laser energy. The delay time and jitter of electric-triggered surface flashover switch [5] are $320 \mathrm{~ns}$ and $50 \mathrm{~ns}$, which are larger than that in the laser-triggered surface flashover experiment.

\section{Conclusions}

Experiment platform of laser-triggered surface flashover is set up, and the delay time and jitter of equipments are measured, respectively. Steady synchronization of the high voltage pulse and laser pulse is obtained. In addition, introductory experiment of laser-triggered surface flashover is carried out using the flat electrodes and columned insulators.

\section{Acknowledgments}

The work was supported by the NSFC (50777061, 50707032 )

\section{References}

[1] G.A. Mesyats, Pulsed Power, Kluwer Academic, New York 2005, p. 151.

[2] H. Bluhm, Pulsed Power Systems, Springer, Berlin 2006, p. 83.

[3] G. Schaefer, Gas Discharge Closing Switch, Plenum, New York 1990, p. 345.

[4] L.M. Earley, G.L. Scott, in: Proc. 8th Pulsed Power Conf., 1991, p. 340.

[5] J.C. Kellogg, J.R. Boiler, Rev. Sci. Instrum. 62, 2689 (1991).

[6] W.C. Nunnally, R. Neurath, in: Proc. 14th Pulsed Power Conf., 2003, p. 1355.

[7] S. Sampayan, G. Caporaso, in: Proc. Particle Accelerator Conf., 1995, p. 2123.

[8] G.J. Caporaso, in: Proc. Int. Linac Conf., 1999, p. 658 .

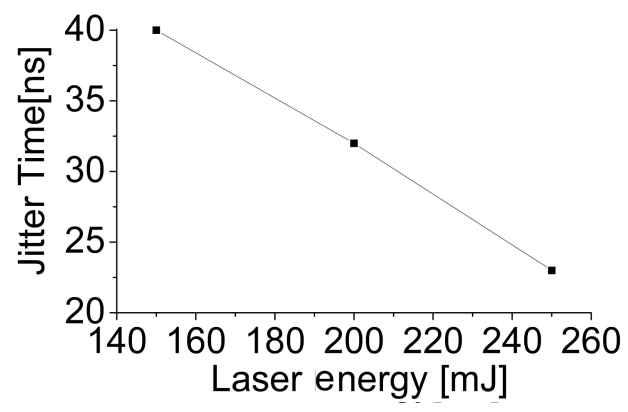

Fig. 9. Jitter time and laser energy. 